Giornale di Tecniche Nefrologiche \& Dialitiche

Anno XIV $n^{\circ} 3$

(C) Wichtig Editore 2002

\title{
L'accesso vascolare: vi è necessità di un'attenzione crescente clinica, chirurgica, diagnostica, tecnica e statistica per una popolazione in dialisi che cresce in età, comorbidità e numero
}

\author{
Giancarlo Ruggieri
}

\section{Dipartimento di Nefrologia e Dialisi, Ospedale San Giacomo in Augusta, Roma}

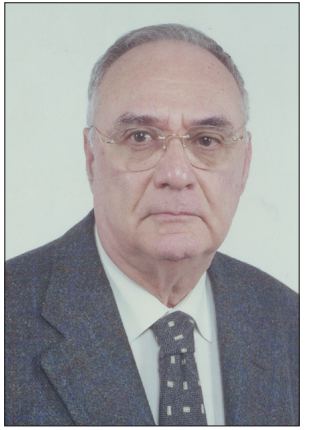

$\int_{n}$ accesso
vascolare, se ben
costruito e di
buone prestazioni
in termini di ac-
cessibilità, flusso
adeguato e conte-
nuto rischio di
infezione e chiu-
sura da trombosi, costituisce la base fondamentale di una valida dialisi extracorporea o, se inadeguato, di un peggioramento della qualità di vita e dell'outcome. Sugli accessi vascolari ci si attenderebbe quindi l'esistenza di un consenso unanime e concretamente vissuto, che tenga dovuto conto sia delle condizioni cliniche che delle varianti metodologiche esistenti in tema di tecnica, di criteri d'uso e di controllo. Ma nella realtà le cose non sono così chiaramente definite, e le soluzioni adottate sono frutto di differenti convinzioni dei nefrologi, che forse non sono sempre inclini a modificarle per mutate esigenze o nuove possibilità, ma anche conseguenza di fattori di vario ambito. Questa premessa appare necessaria alla luce delle mutate esigen- ze osservabili nella popolazione in dialisi, e mi sembra utile esemplificarla ricordando il lungo e complesso passaggio dallo shunt esterno alla fistola arterovenosa.

L'utilizzazione della vena cefalica arterializzata mediante un'anastomosi all'arteria radiale come accesso vascolare venne comunicata da Brescia e Cimino nel 1966, nello stesso anno in cui Ramirez proponeva una versione dello shunt di Scribner e Quinton rettilinea e dotata di alette di stabilizzazione (winged in line); i vantaggi della FAV in termini di sicurezza e libertà personali e di assai minore rischio trombotico vennero apprezzati assai precocemente da vari autori, già nel 1967-69 (alcuni: Hanson et al, Brit Med J - Sperling, che ne propose la versione termino-terminale, Dstch Med Wschr - Menno et al, Trans Am Soc Artif Intern Organs - Lacquet et al, Proc Europe Dial Transpl Ass Patel et al, Brit Med J - Dittrich Hämedialyse und Peritonealdialyse, Springer Verlag), mentre se ne segnalavano i problemi (necessità di usare una pompa sangue, chiusure della FAV subito dopo la confezione, emorragie ed ematomi da puntura, aneuri- smi): ciononostante Buselmeier nel 1972 (sei anni dopo la proposta della FAV di Brescia-Cimino) presentava un modello del tutto nuovo di shunt, giudicato dall' autore "advantageous over the standard Quinton-Scribner shunt and AV fistula", confermandone i vantaggi ancora nel 1974, mentre pubblicazioni dello stesso periodo e durante gli anni '70 dimostravano la crescente adozione della FAV; gli shunts continuavano a presentare un importante, seppur decrescente, utilizzo nel corso degli anni '70, con qualche utilizzo fino ai primi anni '80, seppure limitato ad alcune aree e Centri dialisi. È indubbio che la diffusione di qualsiasi innovazione necessiti tempo sia per le verifiche che per i suoi affinamenti: ciò malgrado, la durata dello shunt esterno per almeno 10 anni dopo il 1966 appare lunga, tenendo conto degli indubbi rilevanti vantaggi della fistola; quindi altre cause hanno variabilmente potuto pesare nel ritardare il passaggio da shunt a fistola: la possibile riluttanza ad abbandonare una tecnica nota per una tecnica diversa e meno conosciuta, la necessità di possedere una sufficiente tecnica di chirurgia vascolare in 
luogo di una procedura abbastanza elementare di cannulazione vasale, il crescente numero di pazienti che allora ricorrevano all'emodialisi a fronte di un numero di nefrologi assai ristretto, e a cui sarebbe spettato anche l'impegno della cannulazione con gli aghi fistola, la necessità di una pompa sangue, uso all'epoca assai limitato nella dialisi con il rene di Kiil. La FAV creava anche un problema in emodialisi domiciliare, molto praticata per l'insufficiente disponibilità dei posti dialisi ospedalieri, con un addestramento reso più difficile con i pazienti portatori di FAV. I criteri di scelta e le relative certezze raggiunte con la FAV sono oggi in qualche modo poste in difficoltà da una realtà clinica diversa e crescente, da cui nuove necessità, sia di ridefinire i criteri di scelta e di controllo degli accessi vascolari, così come di giungere a un miglioramento delle alternative alla FAV tradizionale (gli accessi protesici e i cateteri a permanenza).

Infatti: 1) l'indice di vecchiaia e l'età attesa di sopravvivenza della popolazione generale sono in crescita; 2) cresce quindi progressivamente il numero di soggetti in dialisi in un'età che al momento non ne prevede una concreta possibilità d'uscita con trapianto, ma anche per la carenza di interventi preventivi possibili e che potrebbero limitare l'ingresso in dialisi di una quota parte di soggetti anziani o molto anziani; 3) da dati 2000 del Ministero della Sanità il più alto tasso di morte risulta legato alle malattie su base ischemica (tasso di 12.6 per 10,000 abitanti); 4) da dati 1999 dell'ISTAT, su 100 soggetti della popolazione generale la prevalenza di ipertensione arteriosa, angina pectoris, infarto del miocardio e diabete mellito per i soggetti oltre i 55 anni, a con- fronto con tutta la popolazione, si presenta come indicato nella Tabella I.

In conseguenza, già nel 1998 il 47.4\% dei dializzati aveva un'età superiore a 65 anni (Registro SIN) e per i pazienti incidenti nella stessa fascia d'età la malattia vascolare rappresentava il $42.9 \%$ e il diabete mellito il $21.55 \%$ delle malattie causali diagnosticate. Tenuto conto della frequente associazione di queste condizioni e che in questa fascia d'età e nell'insufficienza renale esse si manifestano in un ambito di malattia vascolare polidistrettuale, ne consegue il dover prendere dovuto atto che in un'importante percentuale della popolazione da dializzare il confezionare fistole arterovenose su vasi nativi e non solo sarà compito più complesso, e che oltre ad avere maggior necessità di studi vascolari preliminari (il che renderebbe ancor più imperativo che opportuno l'avvio precoce al nefrologo), richiederà un affinamento delle tecniche $\mathrm{e}$ delle strategie chirurgiche vascolari, saranno opportuni controlli periodici più frequenti e tecnicamente più impegnativi, vi saranno maggiori occasioni di intervento riparatore e che infine, e malgrado tutto, una FAV potrà non risultare realizzabile in un variabile numero di casi, il che rende imperativo un forte impegno a migliorare il cateterismo permanente.

Se un più elevato livello di attenzione e cura, come ora detto, sarà sempre più necessario, quanto di esso è poi attendibile che si realizzi nella pratica clinica a breve e medio termine, alla luce di quanto finora noto? Goransson e Bergrem nella europea Norvegia (J Intern Med, 2001) hanno osservato che nessuna differenza esisteva fra invio precoce verso tardivo in tre gruppi di pazienti osservati nei perio-

\section{TABELLA I}

\begin{tabular}{lcccc}
\hline Fascia d'età & Ipertensione & $\begin{array}{c}\text { Angina } \\
\text { pectoris }\end{array}$ & Infarto & $\begin{array}{c}\text { Diabete } \\
\text { mellito }\end{array}$ \\
\hline $55-64$ & 24.4 & 1.8 & 2.5 & 7.5 \\
$65-74$ & 33.3 & 3.0 & 3.7 & 11.7 \\
$75->75$ & 37.6 & 4.3 & 4.6 & 12.6 \\
Tutta la popolazione & 9.7 & 0.9 & 1.4 & 3.4 \\
\hline
\end{tabular}

di 1984-88, 1989-93, 1994-98 (in dieci anni), che tutti i pazienti inviati tardivamente erano più vecchi, peggio nutriti e con maggiore terapia farmacologica, in tutti i pazienti il catetere era stato il primo accesso (osservato anche da Astor, Am J Kidney Dis 2001) e vi era stata maggior degenza: osservazioni consimili sono state fatte da altri anche in epoche precedenti ma la situazione non appare cambiata. Un'ampia letteratura ha dimostrato una differenza altamente significativa in durata e numero di interventi fra fistola arterovenosa nativa e protesica, a danno di quest'ultima, fatto rilevato molte volte e a distanza di tempo nello stesso ambiente statunitense (Windus, Am J Kidney Dis 1993 - Gibson et al. Kidney Int, 2001- J Vasc Surg, 2001), ove, malgrado le raccomandazioni delle linee DOQI internazionali, le protesi tuttora dominano la scelta: $\mathrm{i}$ dati normalizzati per i fattori di rischio, riguardanti l'accesso vascolare di 6400 pazienti arruolati nella ricerca URREA - DOPPS, ha confermato clamorosamente sia la rilevante differenza d'uso di FAV, protesi e cateteri fra Europa e USA nei pazienti incidenti (FAV 66\% vs $15 \%$, protesi $2 \%$ vs $24 \%$, cateteri $31 \%$ vs $60 \%$ ) che la maggior durata nel tempo della FAV, sia in assoluto FAV vs protesi che in confronto FAV Europa vs FAV USA (Pisoni et al, Kidney Int, 2002). Dati i risultati, perché FAV protesiche e non da vasi nativi? Il sistema sanitario USA, basato su criteri e sistemi profit, rende largamente ammissibile fra le possibili spiegazioni il profitto degli operatori, superiore con la protesi, scelta che in Europa, dotata in genere di un sistema sanitario fondamentalmente non profit, avrebbe assai meno senso, anche se non credo che il nefrologo ospedaliero italiano o comunque europeo usa poco le protesi solo perché non ci guadagna: è comunque un rischio potenziale, perché è indubbio e provato che, ove divenga forte, la pressione del profitto può orientare le terapie, e non sempre per il meglio: valga per tutto e almeno per l'Italia la forte correlazione inversa che esiste fra prevalenza di Centri profit in un'area e uso della dialisi peritoneale. 
Alcune altre considerazioni pongono ancora dubbi sulla grandezza di probabilità che percorsi clinici "virtuosi" come quelli prima esposti siano già o saranno realmente adottati in modo ubiquitario. Come detto, il crescente ingresso di soggetti con potenziali difficoltà per le FAV da vasi nativi dovrebbe vedere l'adozione a carattere diffuso di maggiori attenzioni prima di ogni approccio operativo e pari accortezze per mantenere attivi gli accessi realizzati. Ogni personale esperienza o convinzione a parte, un'ampia letteratura recente (2000-2001) sottolinea l'indubbia utilità di uno studio vascolare con ultrasuoni e ove necessario radiologico prima di decidere tipo e sede dell'accesso, così come una valutazione periodica del flusso, sia con ultrasuoni che con metodi diluizionali o altri, per diagnosticare e quindi curare precocemente le stenosi; inoltre, l'opportunità di scegliere in modo selettivo se trattare tale patologia con tecniche di radiologia interventistica o di chirurgia, l'importanza del reintervento correttivo precoce nella FAV con inadeguata maturazione e l'utilizzo della microchirurgia: ma eguali segnalazioni sono già state fatte nel corso degli anni (Lawrence, Surgery 1981 - Glanz, An Surg 1987 - May, Kidney Int 1997), senza che evidentemente venissero raccolte, date le "raccomandazioni" degli ultimi lavori e del DOQI; è possibile peraltro che rimborsi "tutto compreso" o difficoltà a reperire le vie per realizzare le procedure suggerite limitino la disponibilità a utilizzarle, anche se dal punto di vista "costo" la somma dei costi delle complicanze non evitate sia in molti casi più elevato del costo delle procedure non eseguite. I cateteri, cui sembra si cominci a ricorrere nella pratica corrente con crescente frequenza, dovrebbero essere l'ultima soluzione, essendo gravati da durata mediamente breve per trombosi irrisolvibili o infezioni foriere anche di gravissime complicanze generali, pur esistendo, come per il catetere peritoneale, un effetto centro, con durate da 10-11 mesi a oltre due anni (Di Iorio et al, Blood Purif 2001 - Jean, Blood Purif 2001) e con indice di infezioni da 1.20 episo$\mathrm{di} / 100 \mathrm{mesi} /$ paziente a oltre 3 episodi, rischi che non sembrano scostarsi molto da quelli della fistola protesica. In definitiva, tenuto conto di quanto detto, mentre la ricerca, clinica, scientifica e industriale dovrebbe cercare, in ogni campo e in collaborazione, $\mathrm{i}$ necessari miglioramenti delle procedure mediche e dei mezzi tecnici, sarebbe con assoluta certezza estremamente utile l'attivazione di un registro di survey permanente e centralizzato (che potrebbe essere affidato allo specifico Gruppo SIN) su ogni aspetto dell' accesso vascolare, costruito sui dati di ogni Centro italiano, non forniti obbligatoriamente ma richiesti formalmente a tutti e con eventuale silenzio-rifiuto a darli, fortemente riservati (in schede a codice segretato, anonime anche per gli osservatori), ma anche disponibili per tutti coloro che li hanno forniti, con regolari elaborazioni comunicate dal Registro a tutti, per consentire a tutti di sapere in termini statistici ciò che avviene, capirlo dai numeri e regolarsi in conseguenza.

giancarlo.ruggieri@virgilio.it 